\title{
Erratum
}

\section{Erratum to: A Penrose-Like Inequality for General Initial Data Sets}

\author{
Marcus A. Khuri ${ }^{\star}$ \\ Department of Mathematics, Stony Brook University, Stony Brook, NY 11794, USA. \\ E-mail: khuri@math.sunysb.edu
}

Received: 4 July 2011 / Accepted: 12 October 2012

Published online: 24 November 2012 - @ S Springer-Verlag Berlin Heidelberg 2012

Commun. Math. Phys. 290, 779-788 (2009)

Two errors in [1] have recently been pointed out by Jan Metzger. The first concerns the constant $\sigma$ in the statement of Theorem 1.2. Namely, this constant is in fact zero. To see this fix large $L>0$. Let $z_{L} \in C^{\infty}(\bar{M})$ be identically 1 up to height $L$ (in $M \times \mathbb{R}$ ), and be zero above height $2 L$, with $\left|\bar{\nabla} z_{L}\right|<2 L^{-1}$. It follows that $\left\|\bar{\nabla} z_{L}\right\|_{L^{2}(\bar{M})} \sim L^{-1 / 2}$. Hence, we find that $\sigma=0$ by letting $L \rightarrow \infty$. The second error concerns Lemma 2.2. Namely, the quantity $\bar{H}-q(\bar{N})$ may not necessarily approach zero as $r \rightarrow 0$. This may be seen by examining certain spherically symmetric examples. Lemma 2.2 is not necessary in order to establish the main result. More precisely, the Penrose-like inequality with a corrected definition of $\sigma$ may be derived from the ideas in this paper, together with some additional arguments. The details will be given by the author in a separate paper.

\section{Reference}

1. Khuri, M.A.: A Penrose-like inequality for general initial data sets. Commun. Math. Phys. 290(2), 779-788 (2009)

Communicated by P. T. Chruściel

^ The author is partially supported by NSF Grant DMS-1007156.

The online version of the original article can be found under doi:10.1007/s00220-009-0830-4. 